Article

\title{
Homo Technologicus: Threat or Opportunity?
}

\author{
Kevin Warwick \\ Office of the Vice-Chancellor, Coventry University, Priory Street, Coventry CV1 5FB, UK; \\ k.warwick@coventry.ac.uk; Tel.: +44-24-7765-9893 \\ Academic Editor: Jordi Vallverdú \\ Received: 29 August 2016; Accepted: 19 October 2016; Published: 26 October 2016
}

\begin{abstract}
Homo sapiens is entering a vital era in which the human-technology link is an inexorable trend. In this paper a look is taken as to how and why this is coming about and what exactly it means for both the posthuman species Homo technologicus and its originator Homo sapiens. Clearly moral and ethical issues are at stake. Different practical experimentation results that relate to the theme are described and the argument is raised as to why and how this can be regarded as a new species. A picture is taken of the status of cyborgs as it stands today but also how this will change in the near future, as the effects of increased technological power have a more dramatic influence. An important ultimate consideration is whether Homo technologicus will act in the best interests of Homo sapiens or not. This paper concludes that the answer is clear.
\end{abstract}

Keywords: cyborgs; implants; posthumans; Homo technologicus; Homo sapiens; human-machine interaction

\section{Introduction}

The subject area of cyborgs and posthumanism has been well developed in the social sciences and humanities for many years now [1]. This has been influenced strongly by the general trend of humans to embrace technology in their everyday lives and depend on it for their existence [2,3]. At the center of the discussion is 'Homo technologicus, a symbiotic creature in which biology and technology intimately interact', with the overall effect that the end result is 'not simply "Homo sapiens plus technology", but the original Homo sapiens morphed by the addition of technology into 'a new evolutionary unit, undergoing a new kind of evolution in a new environment' $[4,5]$.

But we must consider what, in practice, we mean by Homo technologicus. The critical element here is the concept of boundary. As has been pointed out by many researchers, e.g., [6,7], the human brain is affected by the technology around us. It develops over time to interact more efficiently with that technology. However it is perhaps somewhat flippant to suggest [7] that we are therefore all cyborgs, even though there may well be a gradual change in our neural make up over a period as a result of the environment around us. Some have gone further and suggested that a blind man with his cane [8] is a cyborg, on the basis that the cane feeds important information to the man about his local environment. Meanwhile a pair of glasses or a hearing aid for a deaf person could be regarded in the same way. In recent years many researchers in the field of wearable computers have become self-professed cyborgs (e.g., $[9,10])$ whilst, in some instances, having much less interaction with the object worn than the blind man with his cane.

So we have case 1 in which the human body in its entirety remains intact whilst some form of technology is positioned close to or attached to the body for some reason as mentioned. Such a situation includes military examples: infra-red night sight incorporated into weapon sighting systems or voice controlled firing mechanisms in the helmet of a fighter pilot. This case (case 1) could also include those who use their cell phone almost constantly or perhaps even those playing games on a Tablet. It may well be that some researchers wish to define such or all people as cyborgs and it may be that the 
individual brains are gradually modified due to external pressure. However the consideration is that they remain as Homo sapiens and do not come into the category of Homo technologicus. In saying this it is recognised that the technology in question may be for therapeutic purposes, for gain or benefit or it may be simply for pleasure.

On the other hand we have witnessed many intrusions of technology into the human body. As examples hip replacements and heart pacemakers are now relatively frequently encountered. They continue a trend in which technology is readily accepted as being a necessary intrusion. Each of these represent modifications intended to compensate for deficiencies [11]. Even in these instances however the establishment of conceptual limits and boundaries becomes a complex process.

The situation lands up on more difficult terrain when, rather than repairing the ineffective parts of a human body, technology is implanted to enhance normal functioning. The situation where technology is implanted into the body but not into the brain/nervous system, whether it is for therapy or enhancement, we refer to here as case 2. In "A Manifesto for Cyborgs: Science, Technology and Social Feminism in the 1980s" [12], Donna Harraway discussed these issues as the disruption of traditional categories. But why should such entities present an ethical problem?

In each case, although the individual's physical capabilities take on a different form and their abilities are possibly enhanced, their inherent mental state, their consciousness, their perception, has not been altered other than to the extent of itself concluding what the individual might be capable of accomplishing. Where the ethical dilemma appears is in the case when an individual's consciousness is modified by the merging of human and technology. Essentially it is not so much the physical enhancements or repairs that should be our cause for concern but where the nature of an individual is changed in certain ways by the linking of human and technology mental functioning. In the case of a human this means linking technology directly with the human brain or nervous system, rather than by a connection which is either external to the nervous system but internal to the body (case 2) or even one which is external to both (case 1).

Even with technology linked directly to the human brain/nervous system this can be merely for therapeutic purposes as is the situation with deep brain stimulation for Parkinson's disease [13]. This is referred to here as case 3. As a result, Homo technologicus is considered in this paper to be one in which the entity is formed by a human-technology brain/nervous system coupling in which the complete entity goes well beyond the norm in terms of Homo sapiens performance (case 4). Whilst this does refer to a relatively narrow definition with respect to all human-technology possibilities, the arguments that follow are dependent on such a definition.

Connections between technology and the human nervous system not only affect the nature of the individual, raising questions as to the meanings of ' $\mathrm{I}$ ' and 'self' but they also directly influence autonomy. An individual Homo sapiens wearing a pair of glasses, whether they contain a computer or not, remains an autonomous entity. Meanwhile a human whose nervous system is linked directly with a computer not only puts forward their individuality for questioning but also, when the computer is part of a network or at least connected to a network, allows their autonomy to be compromised. It has to be accepted that when this is merely for therapeutic reasons (case 3) there is not, under normal circumstances, an issue. However it is when the individual is enhanced by such an arrangement (case 4) that is the principle subject of this paper.

The main question arising from this discourse being: when an individual's consciousness is based on a part human part technological nervous system/brain, in particular when they exhibit enhanced consciousness, will they hold to the values of Homo technologicus? These being potentially distinctly different to the values of Homo sapiens. Importantly, as a consequence, will such a Homo technologicus entity regard Homo sapiens in a Nietschian way [14], i.e., how humans presently regard cows or chimpanzees?

Some may prefer to look through philosophical pink glasses [12] and see posthumans as being "conducive to the long range survival of humans." But surely it will be those who are members 
of Homo technologicus and not Homo sapiens who will make the pro-Homo sapiens, anti-Homo sapiens decisions.

In this article we look briefly at some case 2 and case 3 practical examples as much to be clear as to which entities are not included in case 4 . We then investigate case 4 in detail and consider some immediate possibilities, given the capabilities of intelligent machines. Finally we consider the future for both Homo technologicus and Homo sapiens. It is not thought to be worthwhile considering case 1 in any further depth than has been done already as this is merely the case of a human holding a pen, riding a bicycle or wearing a watch, no matter how some may wish to market their research $[9,10]$.

\section{Case 2}

In this section we consider different practical instances of implants in the human body, but not in the brain/nervous system, designed more for enhancement purposes rather than for therapy.

\subsection{Identification}

My first electronic implant was a simple radio frequency transmitter, termed a Radio Frequency Identification Device (RFID) which was inserted in my left arm in 1998. As a result the computer in my building allowed me to open doors and switch on lights simply by walking through particular doorways [15]. One interesting feature of the experiment was that I rapidly came to regard the implant as being part of me, something that is a common feature with implantees.

I had the luxury of a doctor to put my implant in place. Many experimenters of today actually carry out their own implantations, learning as they go about basic medicine and sterilisation. In 1998, I had the comfort of local anaesthetic, not so many of today's subjects do, although a friend may well be on hand in case of fainting.

The RFID is perhaps the most common implant tried, recently in the form of a near field communication (NFC) version. This is essentially the same technology as is used in contactless payment cards except that it's now packaged in a small tube about the size of a grain of rice. In 1998 my RFID was almost an inch $(2.54 \mathrm{~cm})$ long. It does require external technology to transmit power to the implant, which has no battery, and to communicate with it.

In recent years many companies have engaged themselves with the technology, although this might be more for publicity than anything else. For example, in January 2015 it was widely reported that several hundred office workers in Sweden had been chipped. With their implants the workers were able to open doors and switch on the photocopier [16].

\subsection{Implant Variety}

The range of possible technology that can be implanted is broad and imaginative. Software developer and biohacker Tim Cannon has experienced a variety of implants. His latest is called the Northstar which lights up under his skin when a magnet is close by. Then there is Lepht Anonym who plans to have a small compass chip implanted near her left knee, along with a power coil that, rather like an RFID, can be charged from an external source.

Meanwhile Moon Ribas has a seismic sensor implanted in her elbow that allows her to feel earthquakes through vibrations, whilst Neil Harbisson, who is otherwise colour blind, has a camera which is attached to his skull. Different colours cause the frequency of vibrations to his skull to vary. As a result he has learned a very high degree of colour discrimination. The technology translates colour frequencies into sound frequencies [17] which are translated into vibrations via an actuator. Initially, Harbisson memorised the frequencies related to each colour, but subsequently he decided to permanently attach the set up to his head.

The project was developed further so that Harbisson was able to perceive colour saturation as well as colour hues. Software was then developed that enabled Harbisson to perceive up to 360 different hues through microtones and saturation through different volume levels [12]. What is particularly interesting about Harbisson's experience is that his discrimination between different colours has 
improved over time as his brain has adjusted to the different vibrations experienced. Clearly the extent of brain adaptability is a pointer to what can be expected in general with regard to either extending the present range of sensory input or rather inputting a complex range of new sensory input information into the human brain that till now has not been possible.

\subsection{Magnet Implants}

One other line of research worth mentioning here is the use of permanent magnet implants for sensory extension. The pads of the middle and ring fingers are the preferred sites for magnet implantation in the experiments that have been reported [18]. The mechanoreceptors in the fingertips are most sensitive to frequencies in the $200-300 \mathrm{~Hz}$ range. An interface containing a coil mounted on a wire frame and wrapped around each finger is used to generate magnetic fields to stimulate magnet movement within the finger. The output from an external sensor is used to control the current in the coil.

Experiments have been carried out in a number of application areas [18]. Ultrasonic range information, involves an ultrasonic sensor for navigation assistance. Distance information from the sensor is encoded as variations in the frequency of pulses. Effectively the closer an external object is to the sensor so the frequency of the pulses increased. The recipient has an accurate indication of how far objects are from the sensor. Further tests have used infrared sensors, which give an indication of the temperature of any objects remotely detected [19]. So the recipient 'feels' the temperature of remote objects.

\subsection{Case 2 Conclusions}

The implants considered in this section certainly, in each case, allow the recipients abilities that are not normal for Homo sapiens. On top of that the recipients regard their implant as being part of their body, which is quite different to something that is merely worn. However, apart from the usual long term brain modification also apparent due to external conditions, there is no immediate change to the neural abilities of the individuals. Hence all those involved in this type of implant are considered to stay within the realms of Homo sapiens with regard to this specific experimentation. In other words, because their implant does not immediately alter their neural make up, the individuals are still considered to be a member of species Homo sapiens.

\section{Case 3}

In this section we look in a little more detail at Case 3 which involves a direct link between the human brain/nervous system and technology, in particular computers and artificial intelligence. However the main point of interest is that the connection is for therapeutic purposes. That said, part of the reason for detailing this section is to see how easy it is for a case 3 example to become case 4 merely by means of software changes.

\subsection{Deep Brain Stimulation}

Deep Brain Stimulation is a procedure employed for the electronic treatment of Parkinson's disease, epilepsy, Tourette's syndrome and clinical depression. The deep brain electrodes can though be connected bi-directionally with a computer such that electrical activity in the brain can be monitored. Ongoing research is developing an 'intelligent' stimulator. This uses artificial intelligence to produce warning signals before Parkinsonian tremors begin [20]. So the stimulator only needs to generate signals occasionally rather than continuously, thus operating in a similar fashion to a heart pacemaker.

Using AI techniques, by better understanding the nature of the disease it has been found that there are distinct types of Parkinson's disease based on the different nature of the electrical activity in the brain [13]. It is also quite possible for the monitoring computer to be located remotely from the patient. Hence, signals within the brain can be tracked in real time and fed into a computer. The computer is 
able to analyse these signals and generate alternative signals that are fed directly back into the brain in order to ensure the person in question continues to function.

Clearly whilst stimulation is provided in a particular part of the brain merely to overcome a specific problem so we are dealing with a case 3 situation. However if the implant is shown to be successful in other ways then the situation could change. As an example when the implant is employed to overcome depression it is an interesting application, giving the individual positive feelings. This could readily be extended for the computer to bring about positive feelings under certain circumstances and negative feelings in other cases.

\subsection{Overcoming Paralysis}

Philip Kennedy developed an operable system which allowed an individual with paralysis to spell words by modulating their brain activity. Kennedy's device used two simple electrodes: the first was implanted in an intact motor cortical region and was used to move a cursor among a group of letters. The second was implanted in a different motor region and was used to indicate that a selection had been made [21].

As the patient thought about moving their fingers these signals were translated into signals to move and stop a computer cursor. The patient could actually see where the cursor was on a large computer screen. Hence they could decide as to when to stop thinking about moving. In this way words could be spelt out letter by letter but also heating and lighting could be controlled quite simply [22].

A different approach was taken by Todd Kuiken whereby nerves normally connected to the pectoralis muscles were employed in a process termed targeted reinnervation. In this procedure, nerves originally connected to arm muscles were reconnected to the pectoralis muscles. As the individual thought about moving their hand and arm so the muscles on the top of their chest flexed instead. External electrodes monitor these movements and send resultant signals to a prosthetic arm worn by the patient. Effectively the person's nervous system is rewired via the pectoralis muscles [23].

The first beneficiary of this technique was Jesse Sullivan, hailed in the media as the world's first 'Bionic Man', who lost both of his arms as a result of an accident he sustained during his work as a high-power electrical lineman. His arms were replaced with robotic prosthetics that he was able to control merely by thinking about using his original arms in the normal way.

\subsection{BrainGate}

The technology which has thus far shown itself to be of the most practical use in this area is the microelectrode array known as the Utah Array, more popularly (and commercially) referred to nowadays as the BrainGate.

The array consists of 100 spikes which are $1.5 \mathrm{~mm}$ long and taper to a tip diameter of less than 90 microns. The spikes, essentially silicon shafts, are arranged in a 10 by 10 array on a $4 \mathrm{~mm} \times 4 \mathrm{~mm}$ substructure and each has a platinum electrode on its tip. The electrodes are linked to platinum wires and in this way the array can be employed bi-directionally to both directly monitor neural activity and also to apply stimulating currents.

A number of trials have been carried out that did not use humans as test subjects, these involving chickens or rats. However it is human studies only that we are more interested in here and these are limited to two groups of studies at the moment. In these experiments the array has been fired into either the human brain or nervous system. In the first set of these experiments to be considered, the array has been employed in a purely recording role for therapeutic results.

Electrical activity from a few neurons monitored by the array electrodes, positioned in the motor cortex, has been decoded into a signal that enabled a severely paralysed individual to position a cursor on a computer screen using neural signals for control in combination with visual feedback. The same technique was later deployed to allow the individual recipient, who was paralysed, to operate a robot 
arm even to the extent of learning to feed themselves in a rudimentary fashion by maintaining sufficient control over the robot arm [24,25].

The same implant was employed to enable a paralysed individual to regain some control over his own arm [26]. In this case signals from the individual's motor cortex were employed to bring about stimulation of hand/wrist muscles via a cuff worn around the person's arm. The effect of this was a sort of bi-pass of the non-functioning nervous system. As a result the individual recipient could make isolated finger movements and perform six different wrist and hand motions.

Initially fMRI scans were taken of the recipient's brain while he tried to copy videos of hand movements. This identified an exact area of the motor cortex dealing with the movements exhibited. Surgery was then performed to implant the array to detect the pattern of electrical activity arising when the recipient thought about moving his hand. These patterns were then sent to a computer which translated the signals into electrical messages, which were in turn transmitted to a flexible sleeve that wrapped around the forearm and stimulated the muscles.

\subsection{Case 3 Conclusions}

We have seen in this section how brain/nervous system to technology connections can be employed to overcome problems such as depression or paralysis. However they have been included here as much to show just how the functioning of the human brain can be altered by the employment of electronic signals. OK here we have considered injection of those signals into specific regions for therapeutic purposes, but obviously other regions and other purposes could be chosen.

\section{Case 4}

In the previous section the BrainGate implant was described and its use was explained in terms of therapeutic procedures. However the same implant has also been employed in experiments aimed at investigating human enhancement beyond the human norm.

\subsection{BrainGate for Enhancement}

In 2002 the BrainGate multi-electrode array was implanted into the median nerve fibers of this paper's author, a healthy human individual, in the course of two hours of neurosurgery to test bidirectional functionality. Stimulation current was applied directly via the implant into the nervous system to allow information to be sent to the recipient, while control signals were decoded from motor neural activity in the region of the electrodes [27].

Overall a number of trials were undertaken successfully using this set up [28].

1 Telegraphic communication directly, electronically between the nervous systems of two humans (the author's wife + the author) was performed.

2 Extended control of a robotic hand across the internet was achieved, with feedback from the robotic fingertips being sent back as neural stimulation for a sense of force by the fingers applied to an object (achieved between the USA and the UK).

3 Extra-sensory (ultrasonic) input was successfully implemented.

4 A wheelchair was successfully driven around by means of neural signals alone. Feedback was in this case purely visual.

5 The color of jewelry was changed as a result of neural signals.

\subsection{Human Enhancement}

In all these cases, the trial could also be described as useful for purely therapeutic reasons, e.g., the ultrasonic sensory input might be of use to an individual who is blind to give them an alternative (bat-like) interaction with the outside world, while telegraphic communication might be beneficial to people with certain forms of motor neuron disease. Each trial can, however, be seen as a potential form of enhancement beyond the human norm for an individual. There was no need to have the implant for 
medical reasons in order to overcome a problem; the experimentation was carried out for the purposes of scientific exploration.

Human enhancement with the aid of brain-computer interfaces introduces all sorts of new technological and intellectual opportunities, but it also throws up different ethical concerns [29,30]. While the vast majority of present day humans are perfectly happy for interfaces, such as the BrainGate, to be used in therapy, the picture is not so clear when it comes to enhancement.

From the trials, it is apparent that extra sensory input is one practical possibility that has been successfully trialed along with extending the human nervous system over the internet. However, improving memory and communication by thought are other distinct potential, yet realistic, benefits with the latter of these also having been investigated to an extent. To be clear these things appear to be possible (from a technical viewpoint at least) for humans in general.

\subsection{Intelligent Machines}

We now have computer technology that, many consider exhibits its own intelligence. As pointed out by Alan Turing [31], this can be considered as being distinct from human intelligence and exhibits a number of different characteristics when compared to human intelligence. Turing said I "May not machines carry out something which ought to be described as thinking but which is very different from what a man does?" In particular a number of positive features associated with the performance of machine intelligence can be picked out and for this section the important thing is to see how the human brain could be dramatically improved in its functioning by a direct link with technology.

As a start "on any issue of computing power, if computers do not have the advantage over human brains already, then they will certainly have it before too long" [32]. This applies to both the speed and accuracy of dealing with data. The biggest advantage of all for machine intelligence however is communication. The present way that humans communicate is extremely poor when compared with that of technology. In speech for example humans convert highly complex electro-chemical signals concerned with emotions, feelings, colours etc. into trivial coded mechanical pressure waves. The possibility of our thoughts remaining in electronic form, as is most likely the case with a machine, would be an enormous step forward for Homo sapiens.

With a human brain linked to a computer brain, that individual could have the ability to [33]:

- $\quad$ use the computer part for rapid maths

- call on an internet knowledge base, quickly

- have memories that they have not originally themselves had

- $\quad$ sense the world in a plethora of ways not possible to Homo sapiens (e.g., ultrasonic, infra-red)

- understand multi dimensionality, as opposed to 3D for the human brain alone

- communicate in parallel, by thought signals alone, i.e., brain to brain

Each one of these examples appears to provide a valid reason as to why for an individual Homo sapiens would wish to upgrade to join the ranks of Homo technologicus.

As for the possibility for machines to have emotions and consciousness then, by following Turing's lead, this is not really an issue. However such characteristics will be very different to those of a human. The big question for research though is to look into the possibilities of integrating human and machine forms of consciousness such that the consciousness of Homo technologicus will be an amalgam of the two. Importantly this form of consciousness, with all its inherent abilities, will almost surely not be comprehensible to Homo sapiens.

\subsection{Case 4 Conclusions}

Case 4 describes the situation when an individual's brain/nervous system is linked directly with a computer for the purposes of enhancement beyond the norm for Homo sapiens. The reason for including this section is to demonstrate that this is a realistic possibility and to assess the sort of enhancements that might well be possible and what this might mean in practice. 


\section{Discussion}

What is the cost for a member of Homo sapiens to become Homo technologicus in the way described and what might the consequences be? Clearly the realisation of such entities presents enormous questions that affect all aspects of human society and culture. In attempting to answer such questions a string of positives and negatives appear. Standing still is not an option. In the extremes, if humans opted by some global agreement for a non-Homo technologicus future (if that were possible), could the end result actually be an intelligent machine superculture as described in [34], leading to the singularity and loss of control on earth to machines. Conversely, if humans globally opted for a Homo technologicus future, could society and culture cope with such a distinct non linearity in evolution?

Some argue that any view of the appearance of superhuman cyborgs can be seen as being unwarranted 'metaphysical' speculation [35]. On the other hand it could be felt that humankind is itself at stake in any case [36]. A viewpoint can then be taken that either it is perfectly acceptable to upgrade humans, turning them into Homo technologicus, with all the enhanced capabilities that this offers, or on the other hand it can be felt that humankind is just fine as it is and should not be so tampered with [36].

The most important issue here is that we are considering a completely different basis on which the Homo technologicus brain operates-part human and part machine in its nature. When the nature of the brain itself is altered the situation is complex and goes far beyond anything encountered with the mere physical extensions of case 1. Such a Homo technologicus entity would have a different foundation on which any of their thoughts would be conceived in the first place. From an individualistic viewpoint therefore, as long as I am myself a Homo technologicus I am happy with the situation. Those who wish to remain Homo sapiens however may not be so happy.

With a brain which is part human, part machine, Homo technologicus would have some links to their human background but their view on life, what is possible and what is not, would be very much different from that of a human. Their values would relate to their own life and Homo sapiens may not figure too highly in such a scenario.

One aspect is that Homo technologicus would have brains, which are not stand alone, but rather, are connected to each other directly via a network. A question is therefore is it acceptable for Homo sapiens to give up their individuality and become mere nodes on an intelligent machine network? Or is it purely a case of individual freedom, if an individual wants to so upgrade then why not?

Some questions are obvious. Should every human have the right to be upgraded? If an individual does not want to should they be allowed to defer, thereby taking on a role in relation to Homo technologicus rather like a chimpanzee's relationship with a human today? How will the values of Homo technologicus relate to those of Homo sapiens? Will Homo sapiens be of any consequence to Homo technologicus other than something of an awkward pain to be removed if possible?

However to conclude, we must be clear that with extra memory, high powered mathematical capabilities, including the ability to conceive in many dimensions, the ability to sense the world in many different ways and, perhaps most importantly of all, communication by thought signals alone, Homo technologicus will be far more powerful, intellectually, than Homo sapiens. It would be difficult imagining that Homo technologicus would want to voluntarily give up their powers or would pay any heed to the trivial utterances of Homo sapiens.

Just as now if a cow enters a room full of humans and proceeds to make cow noises (moo or boo!), it would be extremely unlikely for the humans in the room to say collectively what a wonderful idea the cow has, yes we will all do what the cow wants immediately. No the cow's noises would be simply ignored and she would be removed from the room and shortly killed. So in the future when a Homo sapiens enter a room full of Homo technologicus members and says something like "I don't like what you're doing". It would be extremely unlikely for the Homo technologicus members in the room to say collectively, what a wonderful idea the human has, we will all do what the human wants immediately. No, the human's noises would be simply ignored and they would be removed from the room and shortly killed. 
Conflicts of Interest: The author declares no conflict of interest.

\section{References}

1. Wolfe, C. What Is Posthumanism? University of Minnesota Press: Minneapolis, MN, USA, 2010.

2. Hayles, N. Traumas of code. Crit. Inq. 2006, 33, 136-157. [CrossRef]

3. Scharff, R.C.; Dusek, V. Philosophy of Technology: The Technological Condition: An Anthology; Wiley-Blackwell: Hoboken, NJ, USA, 2003.

4. Longo, G. Body and Technology: Continuity or discontinuity? In Mediating the Human Body: Communication, Technology and Fashion; Fortunati, L., Katz, J., Riccini, R., Eds.; Lawrence Erlbaum: Mahwah, NJ, USA, 2002; pp. 23-30.

5. Callus, I.; Herbrechter, S. Introduction: Posthumanist subjectivities, or, coming after the subject. Crit. Psychol. 2012, 5, 241-264. [CrossRef]

6. Greenfield, S. Mind Change: How Digital Technologies Are Leaving Their Mark on Our Brains; Rider: London, UK, 2015.

7. Clark, A. Natural Born Cyborgs; Oxford University Press: Oxford, UK, 2004.

8. Bateson, G. Steps to an Ecology of Mind; Ballantine Books: New York, NY, USA, 1972.

9. Pentland, A.P. Wearable intelligence. Sci. Am. 1998, 9, 90-95.

10. Mann, S. Wearable Computing: A first step towards personal imaging. Computer 1997, 30, 25-32. [CrossRef]

11. Hayles, N. How We Became Posthuman: Virtual Bodies in Cybernetics, Literature and Informatics; University of Chicago Press: Chicago, IL, USA, 1999.

12. Haraway, D. Simians, Cyborgs and Women; Free Association Books: London, UK, 1996.

13. Cámara, C.; Isasi, P.; Warwick, K.; Ruiz, V.; Aziz, T.; Stein, J.; Bakštein, E. Resting Tremor Classification and Detection in Parkinson's Disease Patients. Biomed. Signal Proc. Control 2015, 16, 88-97. [CrossRef]

14. Nietsche, F. Thus Spoke Zarathustre; Penguin Classics: London, UK, 1961.

15. Warwick, K. The Cyborg Revolution. Nanoethics 2014, 8, 263-273. [CrossRef]

16. Cellan-Jones, R. Office puts chips under staff's skin. Available online: http://www.bbc.co.uk/news/ technology-31042477 (accessed on 17 October 2016).

17. Harbisson, N. Painting by ear. In Modern Painters. The International Contemporary Art Magazine; Louise Blouin Media: New York, NY, USA, 2008; pp. 70-73.

18. Hameed, J.; Harrison, I.; Gasson, M.; Warwick, K. A novel human-machine interface using subdermal implants. In Proceedings of the IEEE 9th International Conference on Cybernetic Intelligent Systems, Reading, UK, 1-2 September 2010; pp. 106-110.

19. Harrison, I. Sensory Enhancement, a Pilot Perceptual Study of Subdermal Magnetic Implants. Ph.D. Thesis, University of Reading, Reading, UK, 2015.

20. Wu, D.; Warwick, K.; Ma, Z.; Gasson, M.; Burgess, J.; Pan, S.; Aziz, T. Prediction of Parkinson's Disease Tremor Onset using Radial Basis Function Neural Network Based on Particle Swarm Optimization. Int. J. Neural Syst. 2010, 20, 109-118. [CrossRef] [PubMed]

21. Kennedy, P.; Bakay, R.; Moore, M.; Adams, K.; Goldwaithe, J. Direct control of a computer from the human central nervous system. IEEE Trans. Rehabil. Eng. 2002, 8, 198-202. [CrossRef]

22. Kennedy, P.; Andreasen, D.; Ehirim, P.; King, B.; Kirby, T.; Mao, H.; Moore, M. Using human extra-cortical local field potentials to control a switch. J. Neural Eng. 2004, 1, 72-77. [CrossRef] [PubMed]

23. Kuiken, T.; Li, G.; Lock, B.; Lipschutz, R.; Miller, L.; Stubblefield, K.; Englehart, K. Targeted muscle reinnervation for realtime myoelectric control of multifunction artificial arms. JAMA 2009, 301, 619-628. [CrossRef] [PubMed]

24. Hochberg, L.; Serruya, M.; Friehs, G.; Mukand, J.; Saleh, M.; Caplan, A.; Branner, A.; Chen, D.; Penn, R.; Donoghue, J. Neuronal ensemble control of prosthetic devices by a human with tetraplegia. Nature 2006, 442, 164-171. [CrossRef] [PubMed]

25. Hochberg, L.; Bacher, D.; Jarosiewicz, B.; Masse, N.; Simeral, J.; Haddadin, S.; Liu, J.; Cash, S.; van der Smagt, P.; Donoghue, J. Reach and grasp by people with tetraplegia using a neurally controlled robotic arm. Nature 2012, 485, 372-375. [CrossRef] [PubMed] 
26. Bouton, C.; Shaikhouni, A.; Annetta, N.; Marcia, A.; Bockbrader Friedenberg, D.; Nielson, D.; Sharma, G.; Sederberg Bradley, P.; Glenn, C.; Mysiw, W.; et al. Restoring cortical control of functional movement in a human with quadriplegia. Nature 2016. [CrossRef] [PubMed]

27. Warwick, K.; Gasson, M.; Hutt, B.; Goodhew, I.; Kyberd, P.; Andrews, B.; Teddy, P.; Shad, A. The application of implant technology for cybernetic systems. Arch. Neurol. 2003, 60, 1369-1373. [CrossRef] [PubMed]

28. Warwick, K.; Gasson, M.; Hutt, B.; Goodhew, I.; Kyberd, P.; Schulzrinne, H.; Wu, X. Thought communication and control: A first step using radiotelegraphy. IEE Proc. Commun. 2004, 151, 185-189. [CrossRef]

29. Warwick, K. Cyborg morals, cyborg values, cyborg ethics. Ethics Inform. Technol. 2003, 5, 131-137. [CrossRef]

30. Warwick, K. Future Issues with Robots and Cyborgs. Stud. Ethics Law Technol. 2010, 4, 6. [CrossRef]

31. Turing, A. Computing Machinery and Intelligence. Mind 1950, 59, 433-460. [CrossRef]

32. Penrose, R. Shadows of the Mind; Oxford University Press: Oxford, UK, 1994.

33. Warwick, K. I, Cyborg; Century: London, UK, 2002.

34. Kurzweil, R. The Singularity Is Near; Gerald Duckworth \& Co. Ltd.: London, UK, 2006.

35. Coole, M. Becoming a cyborg as one of the ends of disembodied man. In Proceedings of the International Conference on Computer Ethics and Philosophical Enquiry, Lancaster, UK, 14-16 December 2001; pp. 49-60.

36. Cerqui, D. The future of humankind in the era of human and computer hybridisation. An Anthropological Analysis. In Proceedings of the Conference on Computer Ethics and Philosophical Enquiry, Lancaster, UK, 14-16 December 2001; pp. 39-48.

(C) 2016 by the author; licensee MDPI, Basel, Switzerland. This article is an open access article distributed under the terms and conditions of the Creative Commons Attribution (CC-BY) license (http://creativecommons.org/licenses/by/4.0/). 